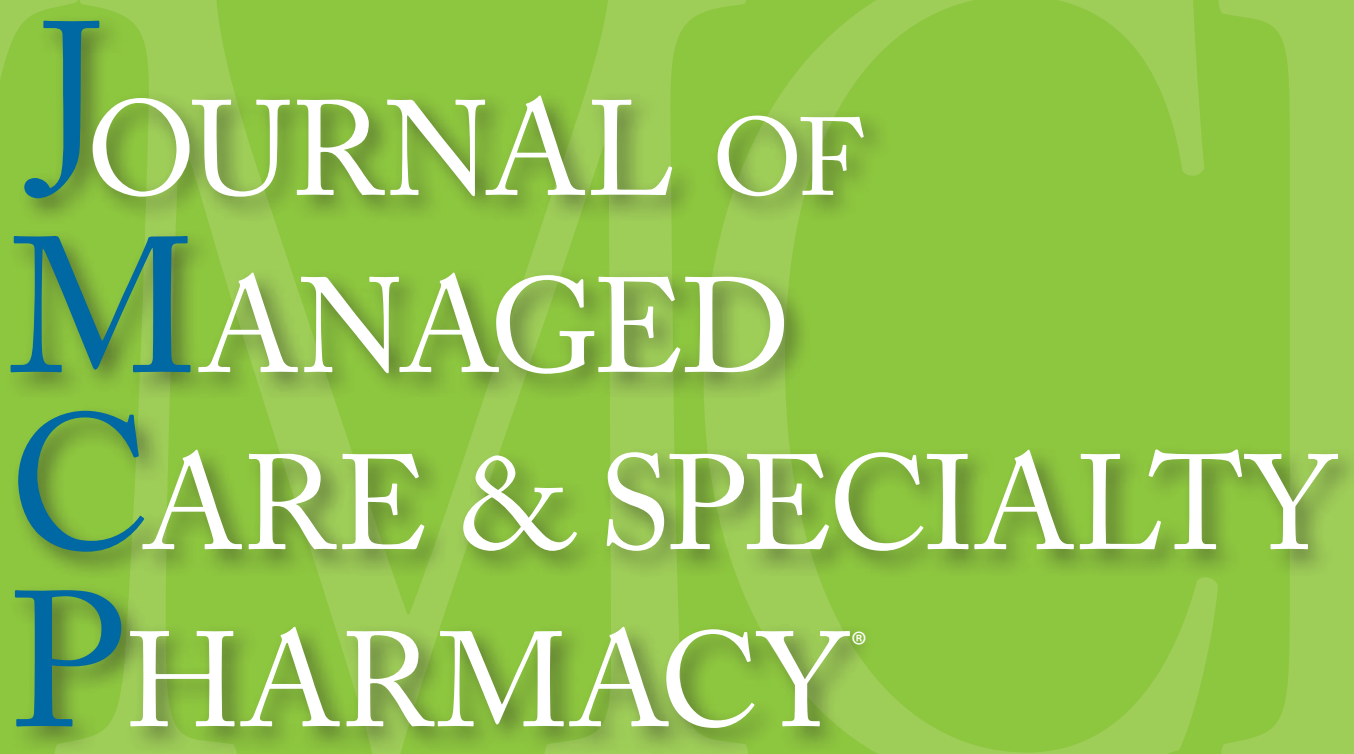

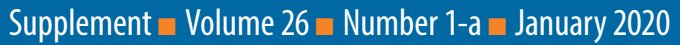

\title{
Direct Costs Incurred Among Women Undergoing Surgical Procedures to Treat Uterine Fibroids
}

Amanda Harrington, PhD; Nicole G. Bonine, PhD, MPH; Erika Banks, MD; Vanessa Shih, PharmD, MS; Dana Stafkey-Mailey, PharmD, PhD; Rupali M. Fuldeore, BAMS, MS; Binglin Yue, MS; Jiatao Michael Ye, MS; Jamie T. Ta, PharmD, MS; and Patrick Gillard, PharmD, MS 


\section{J \\ $\mathrm{MCP}$}

Publisher

Susan A. Cantrell, RPh, CAE

Chief Executive Officer

Academy of Managed Care Pharmacy

\section{Editor-in-Chief}

Laura E. Happe, PharmD, MPH

727.488.2700, lhappe@jmcp.org

\section{Assistant Editors}

Donald G. Klepser, PhD, MBA

University of Nebraska Medical Center, Omaha, NE

Melissa S. McCart, PharmD, MS

Xcenda, Palm Harbor, FL

Karen L. Rascati, PhD

University of Texas College of Pharmacy, Austin, TX

\section{Section Editors}

Carrie McAdam-Marx, BSPharm, MS, PhD

University of Arkansas for Medical Sciences

Little Rock, AR

Michael T. Rupp, BSPharm, PhD

Midwestern University

Glendale, AZ

\section{Managing Editor}

\section{Jennifer A. Booker}

703.317.0725,jmcpreview@amcp.org

\section{Production Editor}

Carol Blumentritt

602.616.7249, cblumentritt@amcp.org

\section{Graphic Designer}

Margie C. Hunter

703.297.9319,mhunter@amcp.org

\section{Account Manager}

Bryan Bonder

American Medical Communications

610.715.4384,Bbonder@americanmedicalcomm.com

Maria Sercia

American Medical Communications

908.216.1548,MSercia@americanmedicalcomm.com

This supplement to the Journal of Managed Care \& Specialty Pharmacy (ISSN 1944-706X) is a publication of the Academy of Managed Care Pharmacy, 675 North Washington Street, Suite 220, Alexandria, VA 22314; 703.684.2600; 703.684.2651 (fax).

Copyright $\odot$ 2020, Academy of Managed Care Pharmacy. All rights reserved. No part of this publication may be reproduced or transmitted in any form or by any means, electronic or mechanical, without written permission from the Academy of Managed Care Pharmacy. POSTMASTER: Send address changes to JMCP,

675 North Washington Street, Suite 220, Alexandria, VA 22314

\section{Supplement Policy Statement}

\section{Standards for Supplements to the} Journal of Managed Care \& Specialty Pharmacy Supplements to the Journal of Managed Care \& Specialty Pharmacy (JMCP) are intended to support medical education and research in areas of clinical practice, health care quality improvement, or efficient administration and delivery of health benefits. The following standards are applied to all JMCP supplements to ensure quality and assist readers in evaluating potential bias and determining alternate explanations for findings and results

1. Disclose the principal sources of funding in a manner that permits easy recognition by the reader.

2. Disclose the existence of all potential conflicts of interest among supplement contributors, including financial or personal bias.

3. Describe all drugs by generic name unless the use of the brand name is necessary to reduce the opportunity for confusion among readers.

4. Identify any off-label (unapproved) use by drug name and specific off-label indication

5. Strive to report subjects of current interest to managed care pharmacists and other managed care professionals

6. Seek and publish content that does not duplicate content in the Journal of Managed Care \& Specialty Pharmacy.

7. Subject all supplements to expert peer review.
AUTHORS

Amanda Harrington, PhD, is an Associate Director of Global Health Economics and Outcomes Research at Allergan, where she leads observational, economic, and clinical outcome assessment studies in support of women's health and central nervous system therapeutic portfolios. She received both a master of science degree and a doctorate in pharmaceutical economics and outcomes research from the University of Arizona College of Pharmacy. During her graduate years, she served as the elected Studen Network Chair for the International Society of Pharmacoeconomics and Outcomes Research. Before her graduate education, she received a bachelor of science in physiology from the University of Arizona.

Nicole G. Bonine, PhD, MPH, is an Associate Director in Health Economics and Outcomes Research (HEOR) with Allergan, where she leads all HEOR in Canada. She received a doctorate in epidemiology and biostatistics and a master's degree in public health, in community health and prevention, from Drexel University in Philadelphia. Before this, she received a bachelor of science in psychobiol ogy from the University of California, Los Angeles. While completing her graduate degree, she led a large population-based study capitalizing on environmental data in an epidemiological sample linking Denmark's health registers. Bonine joined Allergan in 2016, leading 2 products for the antiinfectives franchise, as well as supporting women's health for the launch of Esmya. In her current role, she supports all products within Allergan from marketed to pipeline in Canada. Her projects within Allergan include leading large observational research studies and developing comprehensive economic models. She has published in major peer-reviewed journals such as Pediatrics and Risk Management and Healthcare Policy.

Erika Banks, MD, is a Professor of Obstetrics \& Gynecology and Women's Health (OBGYN); Vice Chair for Education, OBGYN; Residency Program Director, OBGYN; and Director of Gynecology at Montefiore Fibroid Center. Her vast clinical expertise in providing advanced clinical services to women in varied practice settings has yielded interesting research and scholarly output that has had a direct effect on clinical practices that have improved treatment options and outcomes for patients. Furthermore her research and clinical practice at the Montefiore Fibroid Center provides the most vulnerable in New York City with advanced treatment options, including access to clinical trials such as the novel uterine-preserving treatment for uterine fibroids. She received her medical degree from the University of Southern California and completed her obstetrics and gynecology residency at Los Angeles County, University of Southern California, where she continued for an additional year as faculty before joining the Albert Einstein/Montefiore faculty.

Vanessa Shih, PharmD, MS, is an Associate Director of Global Health Economics and Outcomes Research at Allergan. She received her bachelor of science from the University of California, Los Angeles, and her doctor of pharmacy from the University of California, San Francisco. She completed her master of science in pharmacy from the University of Washington.

Dana Stafkey-Mailey, PharmD, PhD, is a Director of the Real World Evidence and Applied Data Analytics team at Xcenda. Stafkey-Mailey has over 15 years of experience leading and conducting HEOR studies for pharmaceutical, biotechnology, and medical device companies. She has extensive experience in the design and analysis of studies using retrospective health care claims data, electronic medical records, customized medical chart abstraction data, cross-sectional and longitudinal survey data, and inpatient care data. Her research has encompassed a wide variety of therapeutic areas, including oncology, hematology, mental health disorders, neurological disorders, infectious diseases, cardiovascular diseases, endocrine disorders, and musculoskeletal disorders. StafkeyMailey has coauthored research published in a variety of peer-reviewed journals, including Annals of Pharmacotherapy, Value in Health, Psychiatric Services, and Journal of Oncology Practice, and regularly presents her work at national and international meetings. Stafkey-Mailey has a doctor of pharmacy from the University of South Carolina, has completed a health outcomes and pharmacoeconomics fellowship at the University of Michigan and Pfizer, and received her doctorate in pharmaceutical economics and policy from the University of Southern California.

Rupali M. Fuldeore, BAMS, MS, is an Epidemiologist and Health Economics Outcomes Researcher at Astellas Global Pharma Development in Northbrook. IL. She received her bachelor's degree in medicine from India and her master of science degree from the Medical College of Wisconsin. Her experience includes generating real-world evidence as Manager at Xcenda and designing and evaluating several disease management programs as Senior Researcher at Anthem. This manuscript is the result of the work she did while employed at Xcenda.

Binglin Yue, MS, is a Biostatistician Manager at Xcenda. She helps pharmaceutical companies to analyze the burden of diseases, treatment patterns, and the health economic outcomes of their products. Yue has strong cancer research and statistical experience using retrospective, prospective, and clinical trial data. Her previous work also includes the assessment and comparison of novel cancer therapies in different clinical trial phases. She has authored more than 20 peer-reviewed articles (including first-authored articles) for such high-impact journals as Lancet Oncology; Nature Communications; Clinical Cancer Research; Cancer Prevention Research; Cancer; International Journal of Radiation Oncology, Biology, Physics; Bone Marrow Transplantation; Journal of Leukocyte Biology; Journal of Urology; Journal of Neuro-Oncology; and Journal of Surgical Oncology. Yue has also served as a reviewer for several medical research journals, including PLoS One; Medicine; Infection, Genetics and Evolution; Journal of Molecular Epidemiology and Evolutionary Genetics of Infectious Diseases; Statistical Methods in Medical Research; and Bioinformatics. Yue received her master of science in biostatistics from the University of Minnesota, Twin Cities.

Jiatao Michael Ye, MS, is a Senior Data Scientist on the Real World Evidence Analytics team at Allergan. He received a master of science in biostatistics from the University of Alabama at Birmingham. Before this, he received a bachelor of science in pharmaceutical science from Wuhan University of China. His main research interests are advanced statistical and machine learning methods, large health care database analysis, image data analysis, and deep learning neural networks.

Jamie T. Ta, PharmD, MS, is a University of Washington/Allergan Global Health Economics and Outcomes Research Fellow. Her experience spans health economics and outcomes research, advanced analytics, specialty pharmacy, corporate development, and market access within the pharmaceutical, managed care, and consulting settings. She earned her bachelor of science and doctor of pharmacy at the University of California, San Diego, and her master of science in pharmaceutical outcomes research and policy at the University of Washington Comparative Health Outcomes, Policy, and Economics (CHOICE) Institute.

Patrick Gillard, PharmD, MS, is an Executive Director of Global Health Economics and Outcomes Research at Allergan. He received his doctor of pharmacy at the University of Washington followed by an master of science in health service research from the CHOICE Institute. 


\section{Table of Contents}

\section{Direct Costs Incurred Among Women Undergoing Surgical Procedures to Treat Uterine Fibroids}

Amanda Harrington, PhD; Nicole G. Bonine, PhD, MPH; Erika Banks, MD; Vanessa Shih, PharmD, MS; Dana Stafkey-Mailey, PharmD, PhD; Rupali M. Fuldeore, BAMS, MS; Binglin Yue, MS; Jiatao Michael Ye, MS; Jamie T. Ta, PharmD, MS; and Patrick Gillard, PharmD, MS

S2 Abstract

S3 Methods

S4 Results

S6 Discussion

S9 Conclusions

S10 References 


\title{
Direct Costs Incurred Among Women Undergoing Surgical Procedures to Treat Uterine Fibroids
}

\author{
Amanda Harrington, PhD; Nicole G. Bonine, PhD, MPH; Erika Banks, MD; Vanessa Shih, PharmD, MS; \\ Dana Stafkey-Mailey, PharmD, PhD; Rupali M. Fuldeore, BAMS, MS; Binglin Yue, MS; \\ Jiatao Michael Ye, MS; Jamie T. Ta, PharmD, MS; and Patrick Gillard, PharmD, MS
}

\begin{abstract}
BACKGROUND: Uterine fibroids (UF) affect up to $70 \%-80 \%$ of women by 50 years of age and represent a substantial economic burden on patients and society. Despite the high costs associated with UF, recent studies on the costs of UF-related surgical treatments remain limited.

OBJECTIVE: To describe the health care resource utilization (HCRU) and allcause costs among women diagnosed with UF who underwent UF-related surgery.

METHODS: Data from the IBM MarketScan Commercial Claims and Encounters database and Medicaid Multi-State database were independently, retrospectively analyzed from January 1,2009 , to December 31 , 2015. Women aged 18-64 years with $\geq 1$ UF claim from January 1, 2010, to December 31,2014 , a claim for a UF-related surgery (hysterectomy, myomectomy, uterine artery embolization [UAE], or ablation) from January 1,2010 , to November 30,2015 , and continuous enrollment for $\geq 1$ year presurgery and $\geq 30$ days postsurgery qualified for study inclusion. A 1-year period before the date of the first UF-related surgical claim after the first UF diagnosis was used to report baseline demographic and clinical characteristics. Surgery characteristics were reported. All-cause HCRU and costs (adjusted to 2017 U.S. dollars) were described by the 14 days pre-, peri-, and 30 days postoperative periods, and independently by the inpatient or outpatient setting.
\end{abstract}

RESULTS: Overall, 113,091 patients were included in this study: commercial database, $n=103,814$; Medicaid database, $n=9,277$. Median time from the initial UF diagnosis to first UF-related surgical procedure was 33 days for the commercial population and 47 days for the Medicaid population. Hysterectomy was the most common UF-related surgery received after UF diagnosis (commercial, $68 \%$ [ $n=70,235]$; Medicaid, $75 \%[n=6,928]$ ). In both populations, $97 \%$ of patients had $\geq 1$ outpatient visit from 14 days presurgery to 30 days postsurgery (commercial, $n=100,402$; Medicaid, $n=9,023$ ), and the majority of all UF-related surgeries occurred in the outpatient setting (commercial, $64 \%[n=66,228]$; Medicaid, $66 \%[n=6,090]$ ). Mean total all-cause costs for patients with UF who underwent any UF-related surgery were $\$ 15,813$ (SD $\$ 13,804$ ) in the commercial population $(n=95,433)$ and $\$ 11,493$ (SD $\$ 26,724)$ in the Medicaid population $(n=4,785)$. Mean total all-cause costs for UF-related surgeries for the commercial/Medicaid populations were $\$ 17,450$ (SD \$13,483)/ $\$ 12,273$ (SD $\$ 19,637$ ) for hysterectomy, $\$ 14,216$ (SD \$16,382)/\$11,764 (SD \$15,478) for myomectomy, \$17,163 (SD \$13,527)/\$12,543 (SD \$23,777) for UAE, $\$ 8,757$ (SD \$9,369)/ $\$ 7,622$ (SD \$50,750) for ablation, and \$12,281 (SD $\$ 10,080) / \$ 5,989$ (SD $\$ 5,617$ ) for myomectomy and ablation. Mean total allcause costs for any UF-related surgery performed in the outpatient setting in the commercial and Medicaid populations were $\$ 14,396$ (SD \$11,466) and $\$ 6,720$ (SD $\$ 10,374)$, respectively, whereas costs in the inpatient setting were $\$ 18,345$ (SD \$16,910) and $\$ 21,805$ (SD \$43,244), respectively.

CONCLUSIONS: This retrospective analysis indicated that surgical treatment options for UF continue to represent a substantial financial burden. This underscores the need for alternative, cost-effective treatments for the management of UF.

J Manag Care Spec Pharm. 2020;26(1-a):S2-S11

Copyright $\odot 2020$, Academy of Managed Care Pharmacy. All rights reserved.
T terine fibroids (UF) are the most common benign
tumors diagnosed in women, affecting up to $70 \%-80 \%$
of women by 50 years of age. ${ }^{1,2}$ While many women with UF often remain asymptomatic, up to 50\% report symptoms including abnormal uterine bleeding, pelvic pressure or pain, and reproductive dysfunction (e.g., obstetric complications, infertility), ${ }^{3-5}$ with $25 \%$ of women with UF experiencing severe symptoms that warrant medical intervention. ${ }^{6}$ Current nonsurgical treatment options for patients with UF are indicated to control symptoms and include off-label or presurgical pharmacologic therapies (e.g., oral, transdermal, and vaginal ring contraceptives; long-acting reversible contraceptive steroids; nonsteroidal anti-inflammatory drugs; tranexamic acid; and gonadotropin-releasing hormone agonists).,5,8 However, as there are few medications currently approved for the management of UF, many women receive surgical intervention; a study of $>84,000$ women diagnosed with UF reported that 39\% had $\geq 1$ UF-related surgical procedure within 1 year following diagnosis. ${ }^{9}$

Common surgical options for the treatment of UF include hysterectomy (uterus removal), myomectomy (fibroid removal), endometrial ablation (uterine lining destruction), and uterine artery embolization (UAE; blocking blood supply to the fibroids). ${ }^{2,10,11}$ Surgical procedures are associated with high costs and risks of surgical complications beyond those of pharmaceutical therapy. ${ }^{12,13}$ A comprehensive insurance claims analysis of 22,860 women who were treated surgically for UF revealed that the majority of women (86\%) undergoing surgery for UF had a hysterectomy. ${ }^{13}$ While hysterectomy remains one of the most common surgical procedures for UF, hysterectomy is not an option for women who want to preserve their fertility. ${ }^{12}$ Procedures that leave the uterus intact present an increased risk of both UF recurrence and future surgery. ${ }^{10,13,14}$

Past studies indicate that costs of UF-related surgical treatments are high and total costs of UF (including treatment and indirect costs) in the United States are estimated at \$5.9-\$34.4 billion annually, representing a substantial economic burden for society and patients. ${ }^{13,15}$ An analysis of a large commercial claims database estimated that the average adjusted costs of a hysterectomy, myomectomy, UAE, and ablation were \$9,562, \$8,969, $\$ 11,114$, and $\$ 4,222$, respectively (2008 U.S. dollars), not including productivity loss. ${ }^{13}$ Despite the significant economic burden imposed by the treatment of UF, recent studies on costs associated with UF-related surgical procedures are limited. 


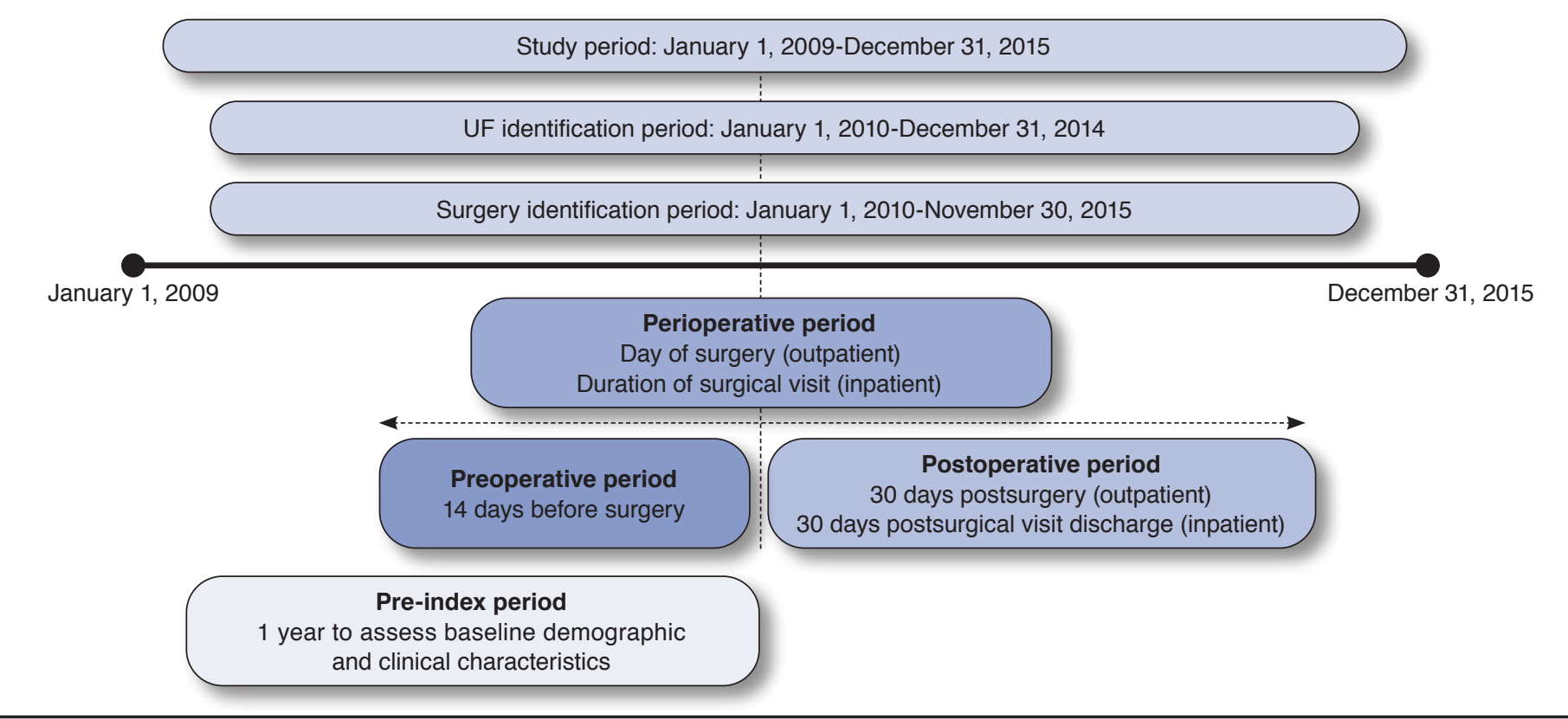

UF = uterine fibroids.

To address the need for up-to-date information, the objective of this analysis was to estimate direct all-cause health care resource utilization (HCRU) and all-cause costs among commercially and Medicaid-insured U.S. women diagnosed with UF who underwent UF-related surgery.

\section{Methods}

\section{Data Sources}

The data sources for this study, analyzed separately, were the IBM MarketScan Commercial Claims and Encounters database and Medicaid Multi-State database. Data between January 1, 2009, and December 31, 2015, were retrospectively analyzed. This study is exempt from institutional review board approval as the data were deidentified.

\section{Study Design and Sample Selection}

A retrospective cohort study was undertaken of women aged 18-64 years with $\geq 1$ UF inpatient or outpatient claim (International Classification of Diseases, Ninth/Tenth Revision, Clinical Modification [ICD-9-CM] codes: 218.X; ICD-10-CM codes: D25.X) between January 1, 2010, and December 31, 2014, and a claim for a UF-related surgery between January 1 , 2010, and November 30, 2015 (Figure 1). UF-related surgeries included hysterectomy, myomectomy, UAE, and ablation. The index date was defined as the date of the first UF-related surgical claim after the first UF diagnosis, and a 1-year pre-index period was used to assess baseline demographic and clinical characteristics. Additional inclusion criteria included continuous health plan enrollment for $\geq 1$ year presurgery and $\geq 30$ days postsurgery. Exclusion criteria included a diagnosis of UF or menopause within 1 year before the date of the first documented claim with a diagnosis of UF, a claim for malignant neoplasm during the entire study period, or $\geq 1$ UF-related surgery on the same day or during a single inpatient visit (except those who received myomectomy and ablation on the same day or during a single inpatient stay).

\section{Surgery Characteristics}

For both the commercial and Medicaid populations, the following surgical characteristics were reported: median (interquartile range [IQR]) number of days from the initial UF diagnosis to the index UF-related surgical intervention; the number and percentage of patients with UF undergoing each specific type of UF-related surgery and subsequent UF-related surgical interventions within 30 days; median (IQR) number of days from the initial UF-related surgical procedure to a secondary UF-related procedure; and the occurrence of types of UF-related surgical procedures in inpatient and outpatient settings.

\section{HCRU and Costs}

All-cause (defined as resource use on all claims regardless of diagnosis) HCRU among patients undergoing UF-related surgery was described for procedures occurring in the 
TABLE $1 \longdiv { \text { Demographic and Clinical Characteristics } }$

\begin{tabular}{|c|c|c|}
\hline Characteristic & $\begin{array}{c}\text { Commercial } \\
\text { Population } \\
(\mathrm{n}=103,814)\end{array}$ & $\begin{array}{l}\text { Medicaid } \\
\text { Population } \\
(\mathrm{n}=9,277)\end{array}$ \\
\hline Mean (SD) age, years & $44.3 \quad(6.4)$ & $41.6 \quad(7.5)$ \\
\hline \multicolumn{3}{|l|}{ Geographic region, n (\%) } \\
\hline Northeast & $13,061(12.6)$ & NR \\
\hline North Central & $22,887(22.1)$ & NR \\
\hline South & $50,939(49.1)$ & NR \\
\hline West & $16,927(16.3)$ & NR \\
\hline \multicolumn{3}{|l|}{ Plan type, n (\%) } \\
\hline $\mathrm{EPO} / \mathrm{PPO}$ & $58,926(56.8)$ & NA \\
\hline $\mathrm{HMO}$ & $20,544(19.8)$ & $3,941(42.5)$ \\
\hline POS & $13,017(12.5)$ & $513(5.5)^{\mathrm{a}}$ \\
\hline CDHP/HDHP & $5,375 \quad(5.2)$ & NA \\
\hline Comprehensive & NA & $4,820(52.0)$ \\
\hline FFS & $2,527 \quad(2.4)$ & NA \\
\hline Unknown & $3,425 \quad(3.3)$ & $3(<1.0)$ \\
\hline Mean (SD) CCI & $0.3 \quad(0.6)$ & $1 \quad(1.5)$ \\
\hline \multicolumn{3}{|l|}{ Specific comorbidities, n (\%) } \\
\hline $\begin{array}{l}\text { Inflammatory disease of the pelvic } \\
\text { organs }\end{array}$ & $10,824(10.4)$ & $4,631 \quad(49.9)$ \\
\hline Depression & $8,638 \quad(8.3)$ & $3,326(35.9)$ \\
\hline Urinary tract infection & $8,348 \quad(8.0)$ & $2,494(26.9)$ \\
\hline Obesity & $7,677 \quad(7.4)$ & $3,058(33.0)$ \\
\hline Endometriosis & $7,396 \quad(7.1)$ & $2,740(29.5)$ \\
\hline Chronic or iron-deficient anemia & $4,762 \quad(4.6)$ & $1,220(13.2)$ \\
\hline Constipation & $2,938 \quad(2.8)$ & $1,255(13.5)$ \\
\hline Infertility & $2,386 \quad(2.3)$ & $46 \quad(0.5)$ \\
\hline Cholelithiasis and cholecystitis & $1,414 \quad(1.4)$ & $313 \quad(3.4)$ \\
\hline \multicolumn{3}{|l|}{ UF-related symptoms, n (\%) } \\
\hline $\begin{array}{l}\text { Vaginal bleeding, menstrual bleeding } \\
\text { disorders }\end{array}$ & $65,911(63.5)$ & $7,620(82.1)$ \\
\hline $\begin{array}{l}\text { Pain associated with female genital } \\
\text { organs }\end{array}$ & $32,585(31.4)$ & $6,089(65.6)$ \\
\hline Urinary symptoms & $6,411 \quad(6.2)$ & $1,229(13.3)$ \\
\hline Anemia due to blood loss & $4,762 \quad(4.6)$ & $1,220(13.2)$ \\
\hline \multicolumn{3}{|c|}{$\begin{array}{l}\text { andicates POS with capitation plan. } \\
\text { bThe CCI, a measure of overall patient health, predicts patient mortality based on } \\
\text { weighted scores of } 1-6 \text { assigned to } 17 \text { conditions. Lower scores (minimum score of } 0 \text { ) } \\
\text { indicate higher predicted survival. } \\
C C I=C h a r l s o n \text { Comorbidity Index; } C D H P=\text { consumer-driven health plan; } \\
E P O=\text { exclusive provider organization; FFS = fee for service; HDHP=high-deduct- } \\
\text { ible health plan; } H M O=\text { health maintenance organization; } N A=\text { not applicable; } \\
N R=\text { not reported; } P O S=\text { point of service; } P P O=\text { preferred provider organization; } \\
S D=\text { standard deviation; } U F=u \text { terine fibroids. }\end{array}$} \\
\hline
\end{tabular}

outpatient (ambulatory surgery, emergency department, physician office, and other outpatient) and inpatient settings, as well as by the 14 days pre-, peri-, and 30 days postoperative periods (Figure 1). For outpatient procedures, the perioperative period included the day of surgery, whereas for inpatient procedures, the perioperative period included the entire length of the inpatient stay. The postoperative period was defined as the 30 days following surgery (excluding day of surgery) for outpatient surgeries and 30 days after discharge (excluding hospital stay) for inpatient surgeries. The number and percentage of patients with $\geq 1$ visit, mean (standard deviation [SD]) number of unique visits, number and percentage of patients with $\geq 1$ pharmacy claim, and mean (SD) number of claims per patient were estimated separately for procedures occurring in inpatient and outpatient settings.

All-cause costs (adjusted to 2017 U.S. dollars), which reflect all payments made to providers of care from both the plan and the patient, were reported. Medical costs were reported by setting of care, and all-cause total costs, defined as the sum of medical and pharmacy costs, were also reported. Patients with evidence of capitated claims anytime during the study period were excluded from cost analyses.

\section{Statistical Analysis}

Descriptive statistics were used to summarize direct all-cause HCRU and costs among patients receiving each type of surgery for all surgical periods. All analyses were performed using SAS version 9.3 (SAS Institute, Cary, NC).

\section{Results}

\section{Patient Demographics and Clinical Characteristics}

Of the 113,091 patients who were included in this study, 103,814 were from the commercial database and 9,277 were from the Medicaid database (Table 1). Medical claims for UF procedures indicated that the majority of patients in both the commercial $(n=94,392$ [91\%]) and Medicaid $(n=7,586$ [82\%]) populations had an associated UF diagnosis on the same claim.

The mean (SD) ages of patients in the commercial and Medicaid groups were 44.3 (6.4) years and 41.6 (7.5) years, respectively (Table 1). The majority of women in the commercial group were enrolled in exclusive provider organization or preferred provider organization plans, whereas the majority of women in the Medicaid group were enrolled in comprehensive plans (Table 1).

Within the baseline year, the most common comorbidity was inflammatory disease of the pelvic organs in both the commercial and Medicaid groups (10.4\% and 49.9\%, respectively), and the most commonly reported UF-related symptoms were vaginal bleeding and menstrual bleeding disorders (63.5\% and $82.1 \%$, respectively; Table 1 ).

\section{Surgery Characteristics}

In the commercial and Medicaid populations, median (IQR) time from the initial UF diagnosis to first UF-related surgical procedure was $33(0,123)$ and $47(0,169)$ days, respectively. Across the surgery types, median (IQR) time to surgery ranged from $9(0,64)$ days for combined myomectomy and ablation to $97(39,311)$ days for UAE in the commercial population, and from $15(0,99)$ days for combined myomectomy and ablation to $139(57,323)$ days for UAE in the Medicaid population. 
TABLE 2 Distribution of Surgery by Type and Place of Service

\begin{tabular}{|c|c|c|c|c|c|}
\hline & Hysterectomy, n (\%) & Myomectomy, n (\%) & UAE, $n(\%)$ & Ablation, n (\%) & $\begin{array}{l}\text { Myomectomy and } \\
\text { Ablation, n (\%) }\end{array}$ \\
\hline & $(\mathrm{n}=70,235)$ & $(\mathrm{n}=15,736)$ & $(\mathrm{n}=3,167)$ & $(\mathrm{n}=12,857)$ & $(\mathrm{n}=1,819)$ \\
\hline \multicolumn{6}{|l|}{ Commercial } \\
\hline Inpatient $(\mathrm{n}=37,586)$ & $32,034 \quad(46)$ & 4,958 & 261 & 190 & 143 \\
\hline \multirow[t]{2}{*}{ Outpatient $(n=66,228)$} & $38,201 \quad(54)$ & $10,778 \quad(68)$ & $2,906 \quad(92)$ & 12,667 & 1,676 \\
\hline & $(\mathrm{n}=6,928)$ & $(n=510)$ & $(n=283)$ & $(\mathrm{n}=1,488)$ & $(n=68)$ \\
\hline \multicolumn{6}{|l|}{ Medicaid } \\
\hline Inpatient $(\mathrm{n}=3,187)$ & 2,954 & $(35)$ & $(7)$ & $(2)$ & $(12)$ \\
\hline Outpatient $(\mathrm{n}=6,090)$ & $3,974 \quad(57)$ & $(65)$ & 264 & 1,463 & (88) \\
\hline
\end{tabular}

Note: Because of rounding, some percentages may not add up to 100.

$U A E=$ uterine artery embolization

The majority of patients with UF who received a UF-related surgical procedure after initial diagnosis had a hysterectomy $(68 \%[\mathrm{n}=70,235]$ in the commercial population and $75 \%$ $[\mathrm{n}=6,928]$ in the Medicaid population; Table 2). The distribution of types of hysterectomy and myomectomy procedures received among women in both populations was as follows: hysterectomy, commercial/Medicaid: 60\%/50\% laparoscopic, $31 \% / 38 \%$ open/abdominal, and $9 \% / 12 \%$ vaginal procedures ( $<1 \%$ of Medicaid patients had an "other" procedure type); myomectomy, commercial/Medicaid: 37\%/35\% hysteroscopy, $34 \% / 35 \%$ abdominal, $26 \% / 19 \%$ laparoscopic, and 3\%/5\% vaginal ( $6 \%$ of Medicaid patients had an "other" procedure type). In the commercial population, myomectomy was the second most common type of UF-related surgery (15\% [ $n=15,736$ ]; Table 2), whereas ablation was the second most common type in the Medicaid population ( $16 \%[n=1,488]$; Table 2$)$. Myomectomy and ablation occurring during the same day was the least common type of UF-related surgical procedure in both the commercial and Medicaid populations (Table 2). Within 30 days of the index procedure, approximately 5\% of patients $(n=4,983)$ in the commercial population and approximately $14 \%(n=1,319)$ in the Medicaid population had a subsequent UF-related surgical procedure, and the median (IQR) time to this procedure was $4(2,7)$ days and $3(2,6)$ days, respectively.

The majority of UF-related surgical procedures occurred in the outpatient setting in both populations (commercial, 64\% [ $n=66,228$ ]; Medicaid, 66\% [ $n=6,090]$; Table 2). A total of $54 \%(n=38,201)$ and $46 \%(n=32,034)$ of hysterectomies occurred in the outpatient and inpatient settings in the commercial population, respectively; and 57\% $(n=3,974)$ and $43 \%$ ( $n=2,954)$ of hysterectomies occurred in the outpatient and inpatient settings in the Medicaid population, respectively. The large majority of all other surgery types took place in the outpatient setting (Table 2).

\section{All-Cause HCRU}

Compared with pharmacy use and inpatient hospitalization, outpatient visits were the most common form of HCRU (Figures 2 and 3). The majority of patients in both populations had $\geq 1$ outpatient claim pre-, peri-, and postsurgery, and 97\% of patients with UF in both the commercial $(n=100,402)$ and Medicaid ( $n=9,023)$ populations had $\geq 1$ outpatient visit (from 14 days presurgery to 30 days postsurgery). The proportion of patients with UF who had $\geq 1$ outpatient visit was $83 \%$ $(n=85,708)$ in the preoperative period and $64 \%(n=65,985)$ in the postoperative period for the commercial population, and 80\% ( $\mathrm{n}=7,444)$ and 79\% $(\mathrm{n}=7,353)$ for the Medicaid population (Figures 2 and 3). The mean number of outpatient visits per patient in the commercial/Medicaid population was 2.0 (SD 1.7)/7.4 (SD 10.1) in the preoperative period and 1.7 (SD 2.1)/9.6 (SD 15.1) in the postoperative period. The proportion of patients with $\geq 1$ pharmacy claim for the commercial and Medicaid populations was $14 \%$ and $53 \%$ in the preoperative period and $64 \%$ and $76 \%$ in the postoperative period, respectively (Figures 2 and 3). The mean number of pharmacy claims per patient for the commercial/Medicaid populations was 0.3 (SD 0.9)/1.8 (SD 2.7) in the preoperative period and 2.0 (SD 2.4)/4.6 (SD 4.8) in the postoperative period. Among patients who had outpatient claims in both populations, the most common type of outpatient visit was "other outpatient" (commercial, $84 \%$ [ $n=83,923]$; Medicaid, 92\% [n=8,270]), followed by laboratory visits (commercial, $79 \%$ [ $n=79,199]$; Medicaid, $82 \%[n=7,382]$ ), and physician office visits (commercial, $79 \%$ [n=79,113]; Medicaid, 74\% [n=6,688]).

The proportion of women with an inpatient hospitalization for UF-related surgical procedures was 36\% and 34\% in the commercial $(n=37,586)$ and Medicaid $(n=3,187)$ populations, respectively. Excluding those who were never hospitalized, the mean (SD) length of hospital stay for women with UF undergoing a UF-related surgical procedure was 3.1 (1.6) days in the commercial population and 2.7 (2.8) days in the Medicaid population. 


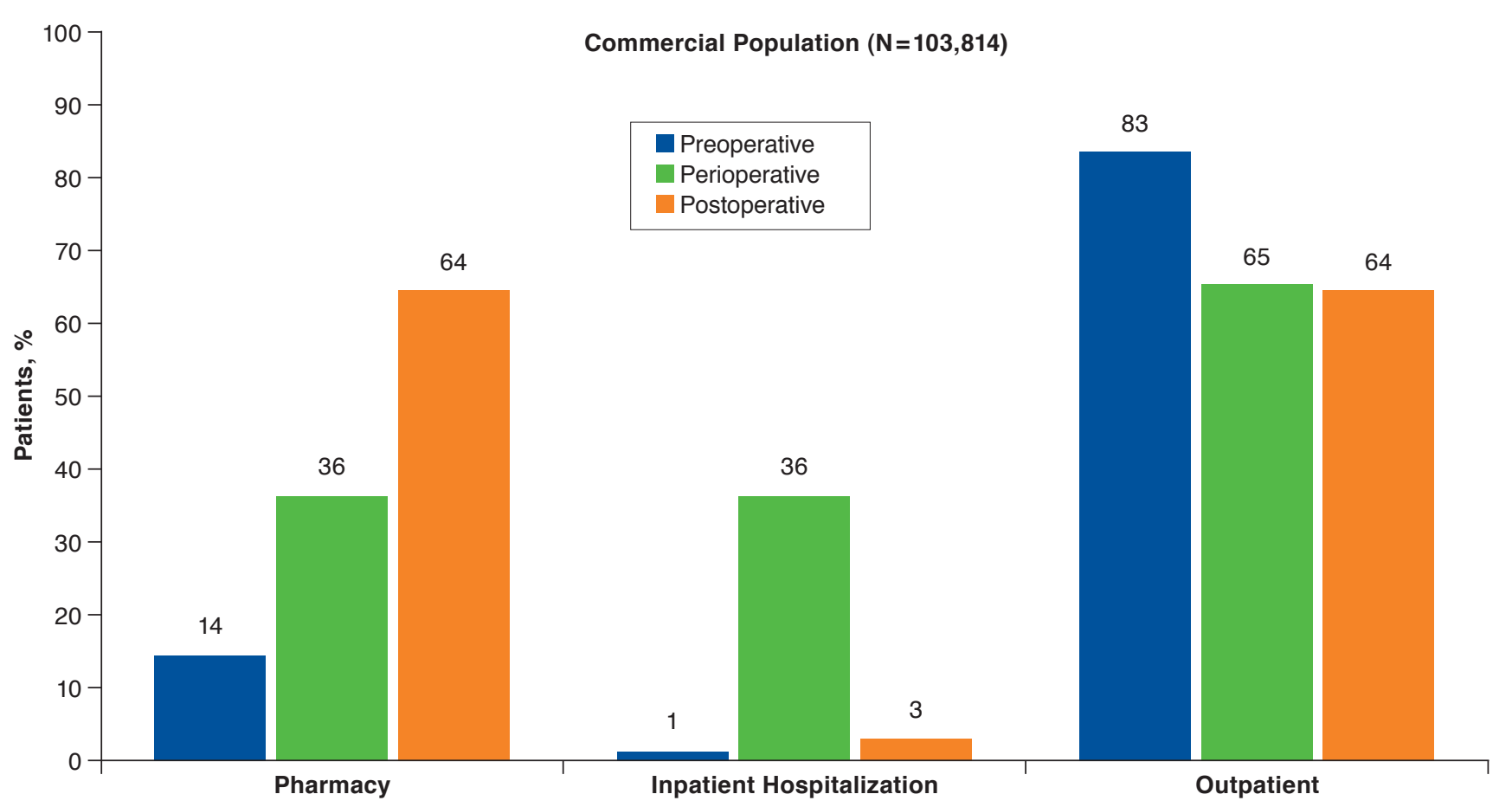

\section{All-Cause Costs}

All-Cause Costs of Surgery. The overall mean total allcause costs (from 14 days presurgery to 30 days postsurgery) for patients with UF who underwent any UF-related surgery were $\$ 15,813$ (SD $\$ 13,804$ ) in the commercial population ( $\mathrm{n}=95,433$ ) and $\$ 11,493$ (SD $\$ 26,724)$ in the Medicaid population $(n=4,785)$. Overall mean total all-cause costs for UF-related surgery procedures in the commercial/Medicaid population were $\$ 17,450$ (SD \$13,483)/ $\$ 12,273$ (SD \$19,637) for hysterectomy, \$14,216 (SD \$16,382)/\$11,764 (SD \$15,478) for myomectomy, \$17,163 (SD \$13,527)/\$12,543 (SD \$23,777) for UAE, $\$ 8,757$ (SD \$9,369)/\$7,622 (SD \$50,750) for ablation, and $\$ 12,281$ (SD $\$ 10,080) / \$ 5,989$ (SD $\$ 5,617$ ) for myomectomy and ablation (Figures 4 and 5).

All-Cause Costs of UF-Related Surgery by Clinical Setting. Mean total all-cause costs for any UF-related surgery performed in the outpatient setting were \$14,396 (SD \$11,466) for the commercial population and $\$ 6,720$ (SD \$10,374) for the Medicaid population, and mean total all-cause costs for any UF-related surgery performed in the inpatient setting were $\$ 18,345$ (SD \$16,910) and $\$ 21,805$ (SD \$43,244) in the commercial and Medicaid population, respectively. Across all surgery types, the mean total all-cause costs for UF-related surgeries performed in the outpatient setting ranged from $\$ 8,500$ (ablation) to $\$ 16,872$ (hysterectomy) in the commercial population and $\$ 5,491$ (ablation) to $\$ 8,346$ (UAE) in the Medicaid population. Mean total all-cause costs for UF-related surgeries performed in the inpatient setting ranged from $\$ 18,157$ (hysterectomy) to $\$ 26,720$ (UAE) in the commercial population and $\$ 4,257$ (myomectomy and ablation) to $\$ 101,940$ (ablation) in the Medicaid population (Figures 4 and 5).

All-Cause Costs of UF-Related Surgery by Period (Pre-, Peri-, and Postoperative). In both the commercial and Medicaid populations, the majority of the mean total direct all-cause costs (79\%95\% and 51\%-95\%, respectively) was attributed to the perioperative period across all surgery types and settings (Figures 4 and 5). Mean total costs of any UF-related surgery by time period in the commercial population were $\$ 1,154$ (SD \$3,793) in the preoperative period, $\$ 13,426$ (SD $\$ 12,121$ ) in the perioperative period, and $\$ 1,233$ (SD $\$ 5,740$ ) in the postoperative period. Mean total costs of any UF-related surgery by time period in the Medicaid population were $\$ 747$ (SD $\$ 2,618$ ) in the preoperative period, $\$ 8,848$ (SD $\$ 24,148$ ) in the perioperative period, and $\$ 1,898$ (SD \$9,264) in the postoperative period.

\section{Discussion}

This retrospective observational study reported direct all-cause HCRU and costs among U.S. women diagnosed with UF who underwent a UF-related surgery to provide an updated overview of the current landscape of surgical treatment options for 


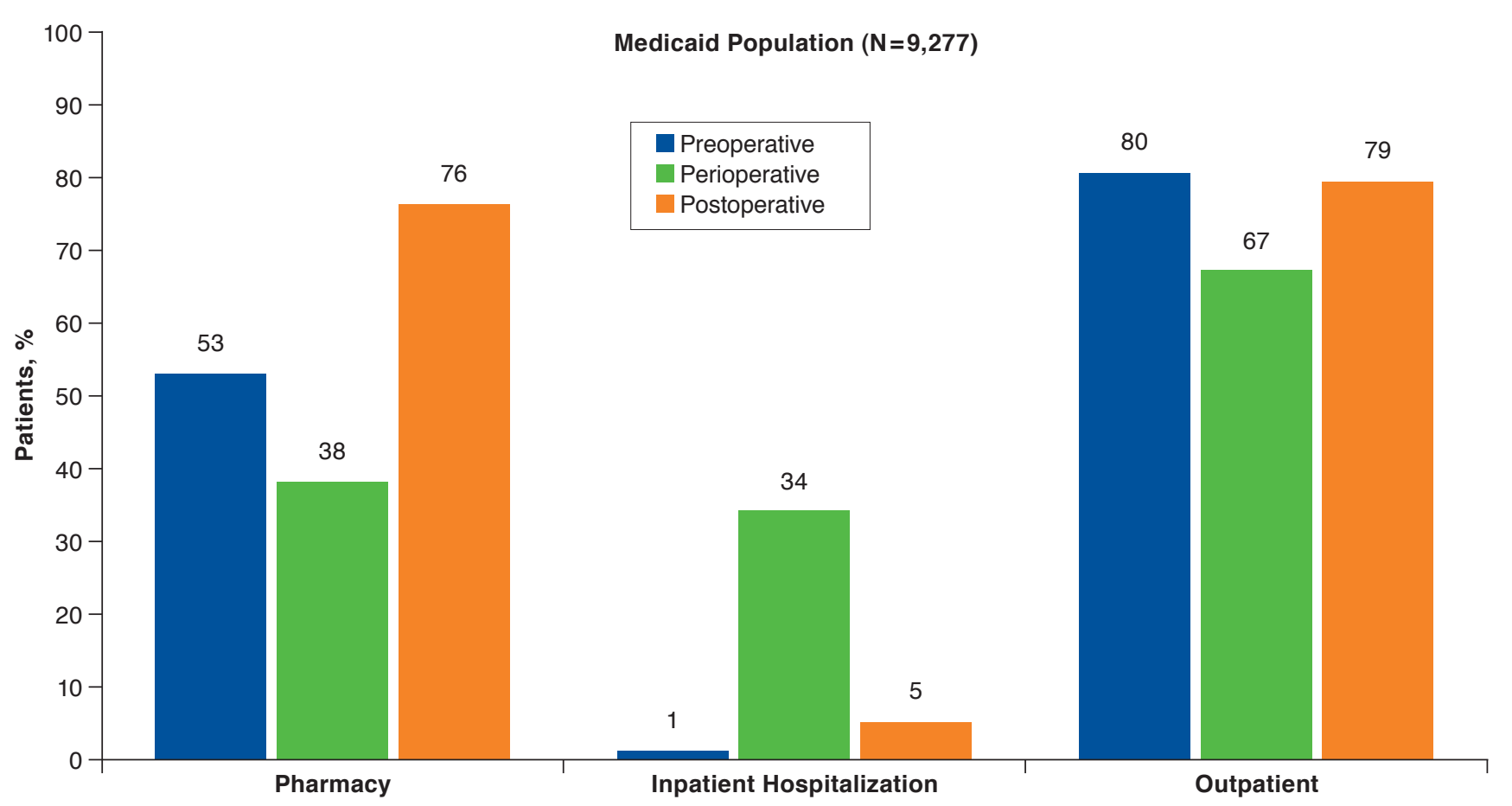

women with UF. In both the commercial and Medicaid populations, median time to occurrence of a UF-related surgical procedure following diagnosis was $<2$ months, with combined myomectomy and ablation and hysterectomy procedures having the shortest median time to surgery. These observations may reflect women's desire to quickly alleviate symptoms, especially those women whose symptoms have considerable effect on their quality of life and daily activities. ${ }^{16}$

Consistent with reports that UF is the most common indication for hysterectomy among reproductive-aged women, ${ }^{5,17}$ more than two thirds of women diagnosed with UF who had a procedure underwent a hysterectomy in both the commercial (68\%) and Medicaid (75\%) populations. However, when compared with the retrospective database analysis by Carls et al. (2008) in which $86 \%$ of women with UF received a hysterectomy, ${ }^{13}$ these results indicate that while hysterectomy continues to be the most common, the percentage of women in the commercial population undergoing a hysterectomy has decreased over time, consistent with published literature. ${ }^{17}$ In addition, the commercial population results further indicate that the percentage of women undergoing myomectomy, UAE, and ablation has increased over time (from $8 \%, 2 \%$, and $5 \%{ }^{13}$ to $15 \%, 3 \%$, and $12 \%$, respectively), indicating that alternative treatment options are increasing in use. The potential reasons for this are numerous and may include concerns regarding the invasiveness of the hysterectomy procedure, costs, a preference for uterine-preserving surgical options, and a desire to retain fertility.

Among commercially insured patients, all-cause costs incurred during the perioperative period were found to be similar to perioperative costs reported in Carls et al., once costs were adjusted to 2017 U.S. dollars. ${ }^{13}$ Furthermore, while hysterectomy occurred at similar rates in the outpatient and inpatient settings, the majority of patients undergoing ablation, myomectomy, UAE, or combined myomectomy and ablation underwent surgery in the outpatient setting. These results are similar to a large study of surgical treatment of UF in the inpatient and outpatient settings across 13 states, though similar myomectomy rates were reported for the inpatient and outpatient settings. ${ }^{11}$ This finding also supports previously reported observations that rates of hysterectomy occurring in the inpatient setting are decreasing over time. ${ }^{11,17}$ Considering that laparoscopic hysterectomy procedures have increased dramatically, and that outpatient hospitalizations generally cost less, it is reasonable to expect this trend to continue. ${ }^{17}$

\section{Limitations}

There are several limitations of this study. Study results were derived from administrative claims data, which may include errors in claims coding and lack relevant clinical data. However, the effect of this limitation would be equally 
FIGURE 4 Mean Total All-Cause Costs by UF Surgery Type, Period, and Setting: Commercial

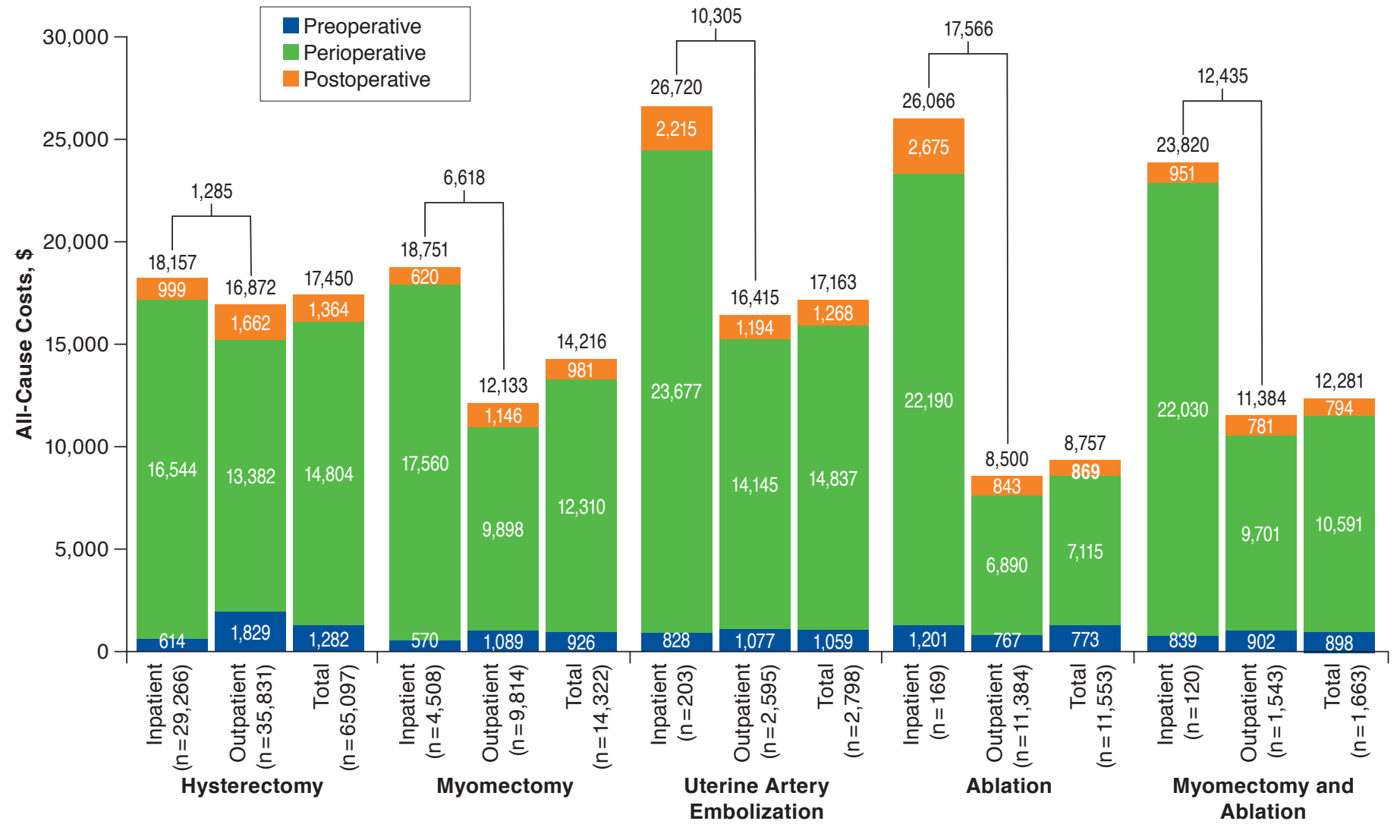

Note: Patients with capitated claims were excluded from cost analyses. Cost differences between inpatient and outpatient procedures are represented by the brackets. Any discrepancies in cost differences are because of rounding during conversion to 2017 U.S. dollars.

$U F=$ uterine fibroids.

distributed across all variables and thus is unlikely to affect 1 outcome more than another. Second, this study was descriptive in nature; therefore, no statistical testing was conducted and potential confounders that may affect health care resource use and costs related to UF treatment were not considered. Further limitations include allocation to a clinical setting based on a hierarchical claims classification, which may have resulted in the misclassification of some of the services to the incorrect setting, as well as possible inclusion of claims unrelated to surgery during the preoperative and postoperative periods. Again, any potential bias caused by this would be equally distributed across all variables and, therefore, would be unlikely to artificially inflate an individual outcome. Additionally, the presence of a diagnosis code on a medical claim does not guarantee positive presence of a disease or symptom, as the diagnosis code may have been incorrectly coded or included as a rule-out criterion. Finally, as with all claims-based analyses, study results may not be generalizable to the overall population, as patients who have commercial or Medicaid health insurance may be different from those without commercial or Medicaid health insurance. However, the large size of the MarketScan claims database, which contains health care data for $>43$ million covered lives, supports the generalizability of these data to a majority of the population. ${ }^{18}$

To test the robustness of the study results in light of the potential limitations described above, subgroup analyses were conducted among commercial patients with and without a UF diagnosis on their index surgery claim. Results indicated that 91\% of patients had a primary diagnosis of UF on their index claim, limiting the possibility that procedures were falsely attributed to UF. In addition, the 14 days presurgery through 30 days postsurgery inclusion period chosen for all-cause costs and HCRU analyses is consistent with previous literature. ${ }^{13}$ 


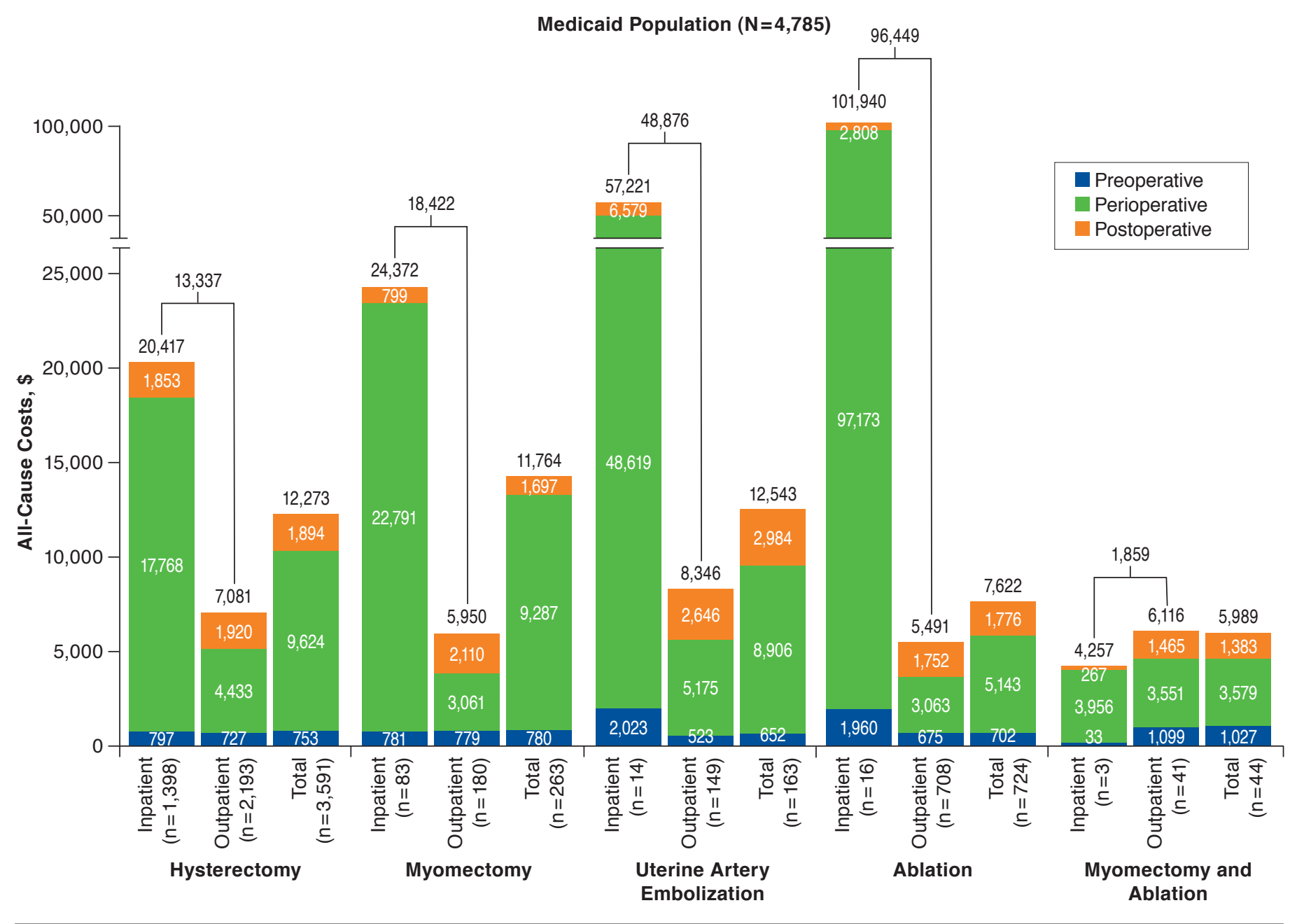

Note: Patients with capitated claims were excluded from cost analyses. Cost differences between inpatient and outpatient procedures are represented by the brackets. Any discrepancies in cost differences are because of rounding during conversion to 2017 U.S. dollars.

$U F=$ uterine fibroids.

In addition, there are several strengths of this study. The sample sizes used to examine women with UF who underwent UF-related surgery are substantial and should provide stable estimates for the variables studied. Much of the existing information about UF among the U.S. population dates back to the early part of the last decade. Therefore, this study serves to update the literature. New surgical techniques and pharmacologic treatment options have continued to increase in number over the past several decades, although the uptake of such treatment and their effect on costs has not been evaluated. Therefore, the information presented here may be timely and valuable in describing the current landscape.

\section{Conclusions}

$\overline{\text { These results emphasize the significant all-cause cost burden }}$ among women undergoing surgical treatment of UF. While hysterectomy is a definitive solution for UF symptoms, it is not an option for women who wish to preserve their fertility or preserve their uterus. Alternative cost-effective treatments would be highly beneficial for patients with UF. The primary objective of UF treatment should be symptom reduction and improvement in quality of life. Thus, the cost of therapy to the health care system and to women with UF must be interpreted in the context of the cost of untreated disease and of ongoing or recurrent symptoms and treatment across intervention modalities. 


\section{Authors}

AMANDA HARRINGTON, PhD, MS; NICOLE G. BONINE, PhD, MPH; VANESSA SHIH, PharmD, MS; and PATRICK GILLARD, PharmD, MS, Allergan, Irvine, California. ERIKA BANKS, MD, Montefiore Medical Center, New York, New York; JIATAO MICHAEL YE, MS, Allergan, Madison, New Jersey; and JAMIE T. TA, PharmD, MS, University of California, San Diego Skaggs School of Pharmacy and Pharmaceutical Sciences, La Jolla. DANA STAFKEY-MAILEY, PharmD, PhD; RUPALI M. FULDEORE, BAMS, MS; and BINGLIN YUE, MS, Xcenda, Palm Harbor, Florida.

AUTHOR CORRESPONDENCE: Amanda Harrington, PhD, Allergan, 2525 Dupont Dr., Irvine, CA 92612. Tel.: 714.246.5666; E-mail: Amanda.Harrington@Allergan.com.

\section{DISCLOSURES}

This study was sponsored by Allergan, Dublin, Ireland. Allergan played a role in the conduct, analysis, interpretation, writing of the report, and decision to publish this study. Harrington and Ye are employees of Allergan. StafkeyMailey, Fuldeore, and Yue are employees of Xcenda. Ta was a contractor at Allergan at the time the study was conducted and is currently supported by a training grant from Allergan. Bonine, Shih, and Gillard are employees of Allergan and have stock, stock options, and/or restricted stock units as employees of Allergan. Banks has no disclosures to report.

This study was presented as a poster at Academy of Managed Care Pharmacy Nexus 2017; October 16-19, 2017; Dallas, TX.

\section{ACKNOWLEDGMENTS}

Writing and editorial assistance was provided to the authors by Patrick Gonyo, PhD, of Complete HealthVizion and funded by Allergan, Dublin, Ireland.

\section{REFERENCES}

1. Baird DD, Dunson DB, Hill MC, Cousins D, Schectman JM. High cumulative incidence of uterine leiomyoma in black and white women: ultrasound evidence. Am J Obstet Gynecol. 2003;188(1):100-07.

2. Donnez J, Dolmans M-M. Uterine fibroid management: from the present to the future. Hum Reprod Update. 2016;22(6):665-86.

3. Stewart EA. Uterine fibroids. Lancet. 2001;357(9252):293-98.
4. Stewart EA. Uterine fibroids. N Engl J Med. 2015;372(17):1646-55.

5. Stewart EA, Laughlin-Tommaso SK, Catherino WH, Lalitkumar S, Gupta D, Vollenhoven B. Uterine fibroids. Nat Rev Dis Primers. 2016:2:16043.

6. National Uterine Fibroids Foundation. About uterine fibroids. Introduction. Updated November 21, 2010. Available at: http://www.nuff. org/health.htm. Accessed December 11, 2019.

7. Duhan N. Current and emerging treatments for uterine myoma-an update. Int J Womens Health. 2011;3:231-41.

8. Yao X, Stewart EA, Laughlin-Tommaso SK, Heien HC, Borah BJ. Medical therapies for heavy menstrual bleeding in women with uterine fibroids: a retrospective analysis of a large commercially insured population in the USA. BJOG. 2017;124(2):322-30.

9. Fuldeore M, Yang H, Soliman AM, Winkel C. Healthcare utilization and costs among women diagnosed with uterine fibroids: a longitudinal evaluation for 5 years pre- and post-diagnosis. Curr Med Res Opin. 2015;31(9):1719-31.

10. Singh SS, Belland L. Contemporary management of uterine fibroids: focus on emerging medical treatments. Curr Med Res Opin. 2015;31(1):1-12.

11. Barrett ML, Weiss AJ, Stocks C, Steiner CA, Myers ER. Procedures to treat benign uterine fibroids in hospital inpatient and hospital-based ambulatory surgery settings, 2013. Agency for Healthcare Research and Quality statistical brief \#200. January 2016. Available at: https://hcup-us.ahrq.gov/ reports/statbriefs/sb200-Procedures-Treat-Uterine-Fibroids.pdf. Accessed December 11, 2019.

12. Soliman AM, Yang H, Du EX, Kelkar SS, Winkel C. The direct and indirect costs of uterine fibroid tumors: a systematic review of the literature between 2000 and 2013. Am J Obstet Gynecol. 2015;213(2):141-60.

13. Carls GS, Lee DW, Ozminkowski RJ, Wang S, Gibson TB, Stewart E. What are the total costs of surgical treatment for uterine fibroids? J Womens Health (Larchmt). 2008;17(7):1119-32.

14. Guo XC, Segars JH. The impact and management of fibroids for fertility: an evidence-based approach. Obstet Gynecol Clin North Am. 2012;39(4):521-33.

15. Cardozo ER, Clark AD, Banks NK, Henne MB, Stegmann BJ, Segars JH. The estimated annual cost of uterine leiomyomata in the United States. Am J Obstet Gynecol. 2012;206(3):211.el-e9.

16. Downes E, Sikirica V, Gilabert-Estelles J, et al. The burden of uterine fibroids in five European countries. Eur J Obstet Gynecol Reprod Biol. 2010;152(1):96-102

17. Morgan DM, Kamdar NS, Swenson CW, Kobernik EK, Sammarco AG, Nallamothu B. Nationwide trends in the utilization of and payments for hysterectomy in the United States among commercially insured women. Am J Obstet Gynecol. 2018;218(4):425.el-e18.

18. IBM MarketScan Research Databases for health services researchers. White paper. April 2019. Available at: https://www.ibm.com/downloads/ cas/6KNYVVQ2. Accessed December 11, 2019. 


\section{Supplement}

\section{Journal of Managed Care \& Specialty Pharmacy

